Review Article

\title{
Research Progress on Anti-Inflammatory Effects and Mechanisms of Alkaloids from Chinese Medical Herbs
}

\author{
Sicong Li $\mathbb{D},{ }^{1}$ Xin Liu $\mathbb{D}^{\circ},{ }^{2}$ Xiaoran Chen, ${ }^{1}$ and Lei $\mathrm{Bi}^{2}$ \\ ${ }^{1}$ School of Pharmacy, Peking University Health Science Centre, Beijing, China \\ ${ }^{2}$ School of Traditional Chinese Medicine, Beijing University of Traditional Chinese Medicine, Beijing 100029, China \\ Correspondence should be addressed to Xin Liu; xinliu1011@126.com
}

Received 8 January 2020; Accepted 17 February 2020; Published 18 March 2020

Guest Editor: Tae Jin lee

Copyright (c) 2020 Sicong Li et al. This is an open access article distributed under the Creative Commons Attribution License, which permits unrestricted use, distribution, and reproduction in any medium, provided the original work is properly cited.

As the spectrum of diseases keeps changing and life pace keeps going faster, the probability and frequency of diseases caused by human inflammatory reactions also keep increasing. How to develop effective anti-inflammatory drugs has become the hotspot of researches. It has been found that alkaloids from Chinese medical herbs have anti-inflammatory, analgesic, antitumor, anticonvulsant, diuretic, and antiarrhythmic effects, among which the anti-inflammatory effect is very prominent and commonly used in the treatment of rheumatoid arthritis, ankylosing spondylitis, and other rheumatic immune diseases, but its mechanism of action has not been well explained. Based on this, this paper will classify alkaloids according to structural types and review the plant sources, applicable diseases, and anti-inflammatory mechanisms of 16 kinds of alkaloids commonly used in clinical treatment, such as berberine, tetrandrine, and stephanine, with the aim of providing a reference for drug researches and clinical applications.

\section{Introduction}

Inflammation has been considered as the main risk factor of rheumatic immune diseases. As the incidence rate of this disease keeps increasing in recent years, how to effectively resist inflammation has attracted widespread attentions. The application of anti-inflammatory Chinese medical herbs not only enjoys a long history, a wide range of sources, and rich varieties, but also has complex and diverse pharmacological effects. Therefore, it has become a research hotspot to search for anti-inflammatory active ingredients in Chinese medical herbs, among which alkaloids are of great representativeness. Alkaloids are nitrogen-containing organic compounds with an alkali like properties. They are widely distributed in Tripterygium wilfordii and Begonia kunmingshanensis of Celastraceae; Sinomenium acutum, Stephania tetrandra S. Moore, and Menispermum dauricum DC. of Menispermaceae [1]; Coptis chinensis Franch., Aconitum carmichaelii, Aconitum kusnezoffii Reichb., Aconitum carmichaelii Debx., and Clematis chinensis Osbeck of Ranunculaceae; Gentiana macrophylla Pall. and Gentiana scabra Bunge. of Gentianaceae, and so on [2].
Some alkaloids have strong anti-inflammatory activities and play important roles in the treatment of rheumatoid arthritis, Behcet's disease, ankylosing spondylitis, myasthenia gravis, systemic lupus erythematosus, dermatomyositis, and other rheumatic immune diseases [3]. The following is a comprehensive review of researches on alkaloids from Chinese medical herbs. By taking chemical structure as the classification standard, anti-inflammatory effects and possible mechanisms of various alkaloids are introduced in detail, which are expected to lay the foundation for follow-up researches and developments of anti-inflammatory drugs.

\section{Isoquinoline Alkaloids}

Isoquinoline alkaloids are widely distributed in 27 sections of plants, such as Menispermaceae, Berberidaceae, Papaveraceae, and Ranunculaceae [4]. Isoquinoline alkaloids, taking isoquinoline or tetrahydroisoquinoline as the basic parent nucleus, can be divided into 20 categories, including simple isoquinoline, benzylisoquinoline, phenethyl isoquinoline, naphthyl isoquinoline, aporphine, morphine, 
phenanthridine, and pyrrolidine. Isoquinoline alkaloids have anti-inflammatory, analgesic, spasmolysis, antibacterial, and relieving asthma effects [5]. Among them, berberine, tetrandrine, dauricine, sinomenine, lycorine, and stephanine have good anti-inflammatory effects.

2.1. Berberine. Berberine, a kind of benzylisoquinoline alkaloid extracted from the rhizome of Coptis chinensis Franch., the bark of Phellodendron chinensis Schneid., and Daemonorops margaritae (Hance) Becc. [6], has therapeutic effects on rheumatoid arthritis, delayed type hypersensitivity, ulcerative colitis, autoimmune tubulointerstitial nephritis, and other rheumatic autoimmune diseases [7]. $8 \mathrm{mg} /$ $\mathrm{kg}$ of berberine subcutaneously injected to rats can inhibit rats' paw swelling induced by carrageenan and mice's auricle swelling induced by xylene. Intraperitoneal injection of berberine $(50 \mathrm{mg} / \mathrm{kg})$ can inhibit the increased skin capillary permeability caused by histamine [8].

Intraperitoneal injection of $30 \mathrm{mg} / \mathrm{kg}$ of berberine for 8 days can inhibit the increased prostaglandin E2 (PGE2) in paw swelling mice induced by formalin [9]. The mechanism is that berberine can inhibit PGE2 synthesis by reducing the concentration of cyclooxygenase-2 (COX-2). Oral administration of berberine $(1.5 \mathrm{mg} / \mathrm{ml})$ can inhibit the delayed hypersensitivity induced by dinitrofluorobenzene in mice [10], by means of inhibiting the production and secretion of interferon- $\gamma$, interleukin-1 (IL-1), tumor necrosis factor (TNF- $\alpha$ ), interleukin-2 (IL-2), interleukin-8 (IL-8), interleukin-6 (IL-6), and monocyte chemotactic protein-1 (MCP1) [11]. Berberine has a therapeutic effect on autoimmune nephritis of rats induced by alloantigens, such as primary anti-glomerular basement membrane and Heymann nephritis. It can inhibit the excretion of urinary protein and reduce serum creatinine content and pathological changes of glomerulus [12].

In terms of diseases in the digestive system, berberine can protect the gastric mucosa from chemical factors. Berberine can inhibit the activation of nuclear factor kappa$\mathrm{B}(\mathrm{NF}-\kappa \mathrm{B})$ and the transcription of inflammatory cytokines in ulcerative colitis mice induced by dextran sodium sulfate [13]. Berberine can inhibit not only the adhesion between endothelial cells and polymorphonuclear leukocytes activated by IL- 1 and tumor necrosis factor- $\alpha$ (TNF- $\alpha$ ) but also the adhesion of polymorphonuclear leukocytes and vascular endothelial cells activated by TNF- $\alpha$, so as to reduce the infiltration of inflammatory cells and lesions of colonic mucosa [14].

2.2. Tetrandrine. Tetrandrine is a kind of bisbenzylisoquinoline alkaloid extracted from the root of Stephania tetrandra S. Moore. and Stephania japonica (Thumb.) Miers [15]. It has therapeutic effects on gout, osteoarthritis, and systemic lupus erythematosus. Tetrandrine can significantly inhibit rats' joint swelling caused by carrageenan or formaldehyde and increased vascular permeability in rats induced by histamine [16]. Intraperitoneal injection of $20 \mathrm{mg} / \mathrm{kg}$ of tetrandrine can significantly reduce the amount of hydrothorax, protein exudation, and leukocyte migration in rats with pleurisy caused by carrageenan [17]. The effect of inhibiting leukocyte migration is stronger than the effect of inhibiting the exudation of hydrothorax and proteins. Intraperitoneal injection of $20 \mathrm{mg} / \mathrm{kg}$ of tetrandrine also significantly reduces the activity of neutrophils (Neu-PLA2) and the acellular component (ACC-PLA2) [18]. Tetrandrine in the treatment of experimental auricular burns plays a direct excitatory adrenal function. Tetrandrine not only inhibits cyclooxygenase (COX)-2 and nitric oxide synthase (iNOS) by interfering with NF- $\kappa \mathrm{B} / \mathrm{COX}-2$ signaling pathway, but also inhibits histamine, platelet-derived growth factor (PDGF), IL-1 $\beta$, and TNF- $\alpha$ in inflammatory reactions and promotes the expression of the anti-inflammatory cytokine IL-10 [19].

Intragastric administration of $6.25 \mathrm{mg} / \mathrm{kg}$ of tetrandrine can reduce the concentration of nitric oxide in serum and pancreatic tissue of rats with acute hemorrhagic necrotizing pancreatitis, reduce the activity of phospholipase A2, and inhibit the activation of NF- $\kappa \mathrm{B}$, thus inhibiting the production of IL-6, IL- 8 , and TNF- $\alpha$ inflammatory factors, as well as reducing the inflammatory response of pancreas [20].

In the inflammatory model of RAW264.7 cells induced by lipopolysaccharide (LPS), tetrandrine can not only inhibit the phosphorylation of NF- $\kappa$ B inhibitor $\alpha$, but also reduce the production of nitric oxide (NO) and prostaglandin E2 (PGE2), and the expression of matrix metalloproteinase-3 (MMP-3) and tissue metalloproteinase inhibitor-1 (TIMP-1) [21].

2.3. Dauricine. Dauricine, a kind of benzylisoquinoline alkaloid extracted from the rhizome and rattan of Menispermum dauricum DC [22], has therapeutic effects on rheumatoid arthritis, ischemic stroke, coronary atherosclerotic heart disease, and ventricular premature beat.

Dauricine can significantly improve neurological deficit symptoms and reduce the apoptosis rate of neurons in ischemic brain tissue [23]. Moreover, it can inhibit the expression of nuclear factor $\mathrm{KB}$, intercellular adhesion molecule-1, and cyclooxygenase-2. Intraperitoneal injection of $40 \mathrm{mg} / \mathrm{kg}$ of dauricine can effectively reduce liver injury in mice induced by $\mathrm{CCl}_{4}$. Its mechanism is related to inhibiting the expression of TGF $\beta 1$ and TNF- $\alpha$ [24].

In vitro studies, it is identified that pretreatment of dauricine can dose-dependently inhibit not only the contents of NO, IL- $1 \beta$, IL- 6 , TNF- $\alpha$, and intercellular adhesion molecule-1 (ICAM-1), but also the activity of inducible nitric oxide synthase (iNOS), myeloperoxidase (MPO), and cyclooxygenase-2 (COX-2) in LPS-stimulated macrophages [25].

Intraperitoneal injection of $50 \mathrm{mg} / \mathrm{kg}$ of dauricine, with pharmacological actions similar to hydrocortisone, can inhibit the increased capillary permeability caused by histamine and acetic acid, inflammatory edema caused by xylene in mice, and edema of hind paws caused by carrageenan in rats [26]. Dauricine has the same anti-inflammatory effect on adrenalectomized rats. The anti-inflammatory effect of dauricine at the same dosage in normal rats is stronger than that in adrenalectomized rats. Dauricine can also reduce the 
content of ascorbic acid in adrenal glands. It can be concluded that dauricine also has an indirect anti-inflammatory effect by stimulating the pituitary adrenal cortex system [27].

2.4. Sinomenine. Sinomenine, a kind of benzyl alkaloid, is extracted from the stem and root of Sinomenium acutum (Thunb.) Rehd. et Wils. [28]. It can be used in the treatment of rheumatoid arthritis, chronic nephritis, ankylosing spondylitis, myocardial ischemia, ventricular premature beats, and other rapid arrhythmias. Sinomenine can inhibit the foot swelling caused by egg white, formaldehyde, or carrageenan [29].

Oral administration of $30 \mathrm{mg} / \mathrm{kg}$ of sinomenine can not only inhibit the activity of iNOS and COX-2 in rats but also inhibit the synthesis and release of PGE2 and leukotriene in inflammatory areas. Sinomenine can alleviate LPS-induced lung inflammation by inhibiting the expression of nitric oxide (NO), myeloperoxidase (MPO), TNF- $\alpha$, and IL-6 [30].

Sinomenine achieves the anti-inflammatory effect by inhibiting NF- $\kappa \mathrm{B}$ and JNK signaling pathways. The mechanism is that it inhibits the expression of TNF- $\alpha$ mRNA and IL- $1 \beta$ mRNA in synovial cells by inhibiting NF- $\kappa$ B activity [31]. Sinomenine can not only inhibit the synthesis of prostaglandin F2a (PGF2a) and leukotriene in macrophages, but also reduce the synthesis of nitric oxide (NO) in cells [32]. Sinomenine can prevent the aggregation of neutrophils caused by complement activation, so as to improve rat antigenic arthritis caused by calf serum albumin. It is found that sinomenine can inhibit the proliferation of lymphocytes induced by mitosis and mixed lymphocyte culture. At the same time, the concentration of IL-1, IL-6, TNF, and other inflammatory cytokines in the supernatant of cultured cells decreases [33]. Moreover, sinomenine has the ability to inhibit inflammation in LPS-stimulated macrophages by regulating CD14/TLR4, JAK2/STAT3 pathway, and calcium signal through alpha $7 \mathrm{nAChR}$ [34].

2.5. Lycorine. Lycorine, a kind of pyrrolidine alkaloid extracted from the bulb of Lycoris radiata (L' Her.) Herb., has a therapeutic effect on osteoarthritis, deformable osteitis, gout, ankylosing spondylitis, and other rheumatic autoimmune diseases. Intravenous injection of $3 \mathrm{mg} / \mathrm{kg}$ of lycorine has a therapeutic effect on formalin arthritis in rabbits and protein arthritis in rats [35]. In the endotoxic shock model mice, $80 \%$ of the mice in normal saline group die of endotoxic shock within 40 hours after injecting LPS. After intravenous injection of $40 \mathrm{mg} / \mathrm{kg}$ of lycorine, the survival rate of mice gets to $60 \%$, rather than $20 \%$ in the normal saline control group [36].

Tolerance ability of inflammatory factors in mice gets improved after injecting lycorine. It is found that lycorine $(5 \mu \mathrm{m})$ can inhibit activities of iNOS and cyclooxygenase- 2 induced by LPS. The inhibitory effect is stronger than that of dexamethasone. In addition, lycorine can also inhibit the production of PGE2 and IL-6 in macrophages to play an antipyretic and analgesic role [37].

A STAT signaling pathway is one of the common pathways of physiological and pathological reactions in the human body. JAK-STAT pathway gets involved in the signal transduction and regulation of many important inflammatory/anti-inflammatory cytokines [38]. STATs are many cytoplasmic proteins that bind to the DNA of the target gene regulatory region. They are conjugated to tyrosine phosphorylation signals to complete transcriptional regulation. It is found that lycorine $(5 \mu \mathrm{M})$ inhibited the activation of STAT1 and STAT3 stimulated by LPS [39].

2.6. Stephanine. Stephanine, a kind of simple isoquinoline alkaloid, is extracted from the root and tuber of Menispermum japonicum Thunb. [40]. It has a therapeutic effect on rheumatoid arthritis, osteoarthritis, and gout. Stephanine has an obvious inhibitory effect on xylene induced inflammatory edema, toe swelling induced by egg white, and adjuvant arthritis. Intraperitoneal injection of $10 \mathrm{mg} / \mathrm{kg}$ of stephanine can reduce myeloperoxidase (MPO) activity and contents of TNF- $\alpha$, IL- $1 \beta$, and IL- 6 in the mammary gland tissue of mastitis rats induced by LPS [41]. Stephanine at this dosage can not only inhibit p65 phosphorylation and $\mathrm{I} \kappa \mathrm{B}$ degradation, but also improve the pathological changes of breast tissue. Intraperitoneal injection of $20 \mathrm{mg} / \mathrm{kg}$ of stephanine can reduce the expression of NF- $\kappa \mathrm{B}$, phospho-p38 MAPK, phospho-JNK, NLRP3, and IL- $1 \beta$ in the brain tissue of mice with focal cerebral ischemia, so as to play an antiinflammatory role [42]. Intragastric administration of $15 \mathrm{~g} /$ $\mathrm{kg}$ of stephanine can not only reduce the content of ICAM-1, IL- $1 \beta$, and IL-18 but also increase the content of IL-12 in peripheral blood and nasal lavage fluid [43].

\section{Piperidine Alkaloids}

Piperidine alkaloids are derived from lysine, including piperidine, indolicidin, and quinolizidine. These alkaloids can be found in Sophora flavescens Ait., Diphasiastrum veitchii., Piper nigrum L., Lobelia chinensis Lour., etc. [44]. Piperidine alkaloids have anti-inflammatory, anticonvulsant, anticancer, and antiarrhythmic effects. Among them, aloperine, sophoridine, and matrine have good anti-inflammatory effects [1].

3.1. Aloperine. Aloperine, extracted from Sophora alopecuroides, has therapeutic effects on myocardial ischemiareperfusion injury, acute renal injury, asthma, ventricular hypertrophy, pulmonary hypertension, and other cardiovascular diseases [45]. The concentration of IL-6, TNF- $\alpha$, and IL- $1 \beta$ in $\mathrm{H} 9 \mathrm{c} 2$ cardiomyocytes decreases significantly after intragastric administration of $50 \mathrm{mg} / \mathrm{L}$ of aloperine for 48 hours, which suggests that aloperine can alleviate myocardial ischemia-reperfusion injury by inhibiting inflammatory response [46]. Intragastric administration of $40 \mathrm{mg} /$ $\mathrm{kg}$ of aloperine for 14 days can reduce the levels of IL-6, IL$1 \beta$, TNF- $\alpha$, NO, MCP-1, ADMA, ICAM-1, and VCAM-1, thus inhibiting the myocardial fibrosis and ventricular hypertrophy caused by isoproterenol [47].

IL- 6 and TGF- $\beta 1$ play an important role in the development of pulmonary hypertension. Pulmonary hypertension is often accompanied by a large number of inflammatory cells 
infiltration into pulmonary vascular lesions. The expression levels of NF- $\kappa \mathrm{B}, \mathrm{TNF}-\alpha$, and IL- $1 \beta$ protein significantly reduce after intragastric administration of $100 \mathrm{mg} / \mathrm{kg}$ of aloperine [48]. The result of flow cytometry shows that $0.5 \mathrm{mM}$ of aloperine can reduce the expression levels of NF$\kappa, \mathrm{Bp} 65, \mathrm{p}-\mathrm{IKK} \alpha, \mathrm{p}-\mathrm{I} \kappa \mathrm{B} \alpha, \mathrm{TNF}-\alpha$, and CyclinE1 [49]. After the application of aloperine, the injury of pulmonary vascular endothelial cells gets significantly improved, the shape of the nucleus of endothelial cells becomes basically normal, and the expansion of the Golgi body and endoplasmic reticulum gets improved. It suggests that rotenone can improve pulmonary hypertension induced by monocrotaline through the NF- $\kappa \mathrm{B}$ p65 signaling pathway [50].

In terms of respiratory diseases, intragastric administration of $40 \mathrm{mg} / \mathrm{kg}$ of aloperine can improve lung function, alleviate asthma symptoms, and inhibit inflammatory responses in asthmatic mice. The mechanism is related to the regulation of the $\mathrm{NF}-\kappa \mathrm{B}$ inflammatory signal pathway and the reduction of TNF- $\alpha$ and IL- $1 \beta$ levels [51].

3.2. Sophoridine. Sophoridine, extracted from the roots of Sophora flavescens, has therapeutic effects on rheumatoid arthritis and ankylosing spondylitis, as well as organ damage caused by endotoxin and exogenous toxicant [52]. Intragastric administration of $40 \mathrm{mg} / \mathrm{kg}$ of sophoridine can reduce the increased permeability of capillaries and inhibit the auricle swelling induced by xylene [53]. Intraperitoneal injection of $5 \mathrm{mg} / \mathrm{kg}$ of sophoridine for 3 days in advance can prevent the lung and kidney injury caused by endotoxin and reduce the congestion, edema, and blood seepage into lung tissue as well as edema and inflammatory cell infiltration in renal tubular epithelial cells [54]. Its anti-inflammatory mechanism is to downregulate the expression of lipopolysaccharide recognition receptor, lipopolysaccharide-binding protein, CD14, and TLR4, as well as the transcription of nuclear factor c-Jun and c-fos genes. Sophoridine inhibits not only the phosphorylation of p38 mitogen-activated protein kinase (p38 MAPK) but also the expression and release of IL-6, TNF- $\alpha$, and NO [55]. Continuously intraperitoneal injection of $125 \mathrm{mg} / \mathrm{kg}$ of sophoridine for 8 weeks significantly inhibits acute liver injury in mice induced by carbon tetrachloride [56]. The damage of liver tissue in mice gets alleviated, which appears as the orderly arrangement of hepatocytes, the reduction of focal necrosis, cell balloon degeneration, steatosis, and interstitial inflammation. The protective mechanism of sophoridine is related to its inhibition of cyclooxygenase- 2 activity and the reduction of TNF- $\alpha$ and other inflammatory cytokines [57].

3.3. Matrine. Matrine, extracted from the root of Sophora flavescens, has therapeutic effects on rheumatoid arthritis, psoriasis, ulcerative colitis, ankylosing spondylitis, asthma, acute lung injury, acute respiratory distress syndrome, and cerebral infarction [58].

Hypodermic injection of $25 \mathrm{mg} / \mathrm{kg}$ of marine can inhibit the swelling of auricle caused by croton oil, the acute inflammatory swelling of the toes caused by carrageenan, or egg white [59]. Moreover, it can inhibit the increased capillary permeability in the abdominal cavity caused by acetic acid in both normal mice and adrenalectomized mice.

In terms of respiratory diseases, 3 hours after endotoxin injection, intraperitoneal injection of marine $(10 \mathrm{mg} / \mathrm{kg})$ can resist the pathological changes of lung tissue in mice caused by lipopolysaccharide and reduce pulmonary edema, pulmonary vascular leakage, and rise in temperature [60]. Moreover, it can not only inhibit the activity of myeloperoxidase (MPO) and malondialdehyde (MDA) but also reduce the content of TNF- $\alpha$, IL- $1 \beta$, and IL- 6 . Matrine can inhibit airway inflammation by inhibiting the signal transduction of NF- $\kappa \mathrm{B}$ in airway epithelial cells of asthmatic mice, reducing the expression of SOCS3 as well as the production of reactive oxygen species (ROS) and inflammatory factors in alveolar macrophages [61].

Matrine also has a protective effect on lung injury caused by ulcerative colitis. The mechanism is to enhance the antiinflammatory and scavenging oxygen free radical ability of lung tissue and upregulate the repair factors in lung and gut tissue by increasing the expression of ZO-1 and Occludin, which are closely related to the mucosa of lung tissue. Matrine has a strong inhibitory effect on TNF- $\alpha$ and can effectively reduce the secretion of IL-1 $\alpha$ and IL-8 [62].

In terms of nervous system diseases, injecting matrine $(30 \mathrm{mg} / \mathrm{kg})$ into the jugular vein can inhibit the activity of $12 /$ 15 -lipoxygenase and the expression of NF- $\kappa \mathrm{B}$, TLR4, and TLR2 in ischemic brain tissue [63]. Moreover, it can reduce not only the nerve function defect of right middle cerebral artery occlusion model rats but also the volume of local cerebral infarction. The results above suggest that matrine has an obvious neuroprotective effect on ischemic brain tissues.

In vitro, matrine at the dosage of $50 \mu \mathrm{mol} / \mathrm{L}$ can significantly inhibit the expression of IL-6, IL-8, TNF- $\alpha$, NF$\kappa \mathrm{B}$, and intracellular adhesion molecule 1 in colon epithelial cells induced by lipopolysaccharide. Matrine at the dosage of $100 \mu \mathrm{mol} / \mathrm{L}$ can significantly resist the inflammatory damage of colon epithelial cells induced by lipopolysaccharide [64].

\section{Terpene Alkaloids}

Terpene alkaloids come from mevalonic acid. They can be classified into four categories, which include monoterpenes, sesquiterpenes, diterpenes, and triterpenes. Terpene alkaloids have antihypertensive, analgesic, anti-inflammatory, antipyretic, and sedative effects. Among them, gentianine, aconitine, and bulleyaconitine A have good anti-inflammatory effects [65].

4.1. Gentianine. Gentianine, a kind of monoterpene alkaloid, is extracted from the root of Gentiana scabra Bunge., the whole herb of Gentiana algida Pall., and the seeds from Trigonella foenum-graecum. Intraperitoneal injection of $90 \mathrm{mg} / \mathrm{kg}$ of gentianine for 10 days can reduce not only the increase of capillary permeability caused by egg white but also the formaldehyde-induced foot swelling in rats, accelerating the swelling to subside as well [66]. The effect is equivalent to $200 \mathrm{mg} / \mathrm{kg}$ of sodium salicylate but does not 
lead to gastrointestinal bleeding [67]. The content of vitamin $\mathrm{C}$ in adrenal gland of rats significantly decreases after injecting gentianine. But this effect disappears after the removal of the pituitary gland. Therefore, it can be concluded that the anti-inflammatory effect of gentianine is related to hypophyseal-adrenocortical functions.

Gentianine inhibits IL- $1 \beta$-induced inflammatory responses in rats' articular chondrocytes by inhibiting the activity of P38, extracellular regulated protein kinases (ERK), and c-Jun N-terminal kinase (JNK). In addition, gentianine can also inhibit the release of matrix metalloproteinases (MMPs) induced by IL-1 $\beta$ and promote the expression of type II collagen [68]. Oral administration of $50 \mathrm{mg} / \mathrm{kg}$ of gentianine for 12 weeks can reduce body weight and visceral fat mass in mice, which was associated with reduced levels of inflammatory cytokines (NF- $\kappa \mathrm{b} 1, \mathrm{TNF}-\alpha$, and IL-6). Oral administration of gentianine at the dosage of $60 \mathrm{mg} / \mathrm{kg}$ for 7 days has a therapeutic effect on contact dermatitis induced by 1-fluoro-2,4-dinitrofluorobenzene (DNFB) [69]. Gentianine can inhibit epidermal hyperplasia and immune cell infiltration, as well as reducing the production of TNF- $\alpha$, IFN- $\alpha$, IL- 6 , and MCP- 1 in inflammatory tissues.

4.2. Aconitine. Aconitine, a kind of diterpene alkaloids extracted from the root tuber of Aconitum carmichaeli Debx., has a therapeutic effect on rheumatoid arthritis, ankylosing spondylitis, and other autoimmune diseases [70]. Oral administration, subcutaneous injection, or intramuscular injection of aconitine can significantly reduce the content of ascorbic acid in the adrenal glands of rats. This effect cannot be blocked by pentobarbital and chlorpromazine. Oral administration of $0.1 \mathrm{mg} / \mathrm{kg}$ of aconitine has inhibitory effects on the edema of toes in rats caused by carrageenan, ear swelling in mice caused by croton oil, and joint swelling in rats caused by formaldehyde [71]. The effect of aconitine exerted on the symptoms above is stronger than indometacin. Intragastric administration of $60 \mathrm{mg} / \mathrm{L}$ of aconitine can improve the acute lung injury in rats caused by lipopolysaccharide, by means of inhibiting the activation of $\mathrm{NF}-\kappa \mathrm{B}$, reducing the contents of inflammation transcription factor NF- $\kappa$ B and inflammatory mediators TNF- $\alpha$, IL- 6 , and IL-1 $\beta$. Moreover, no evidence has been founded to support aconitine's toxicity to the cells of the lung tissue [72].

4.3. Bulleyaconitine A. Bulleyaconitine A, a kind of aconitine diterpenoid alkaloids, is extracted from the root tuber of Aconitum kusnezoffii Rchb. [73]. Nowadays, bulleyaconitine A has been widely used in the clinical treatment of rheumatoid arthritis, ankylosing spondylitis, knee osteoarthritis, and gout. Bulleyaconitine $\mathrm{A}$ has an obvious anti-inflammatory effect by inhibiting the phagocytic function of macrophages as well as the release of nitric oxide, INF- $\gamma$, and PGE2 [74]. Intraperitoneal injection of $0.48 \mathrm{mg} / \mathrm{kg}$ for 7 consecutive days can effectively reduce the content of IL-4, IL-10, IL-6, TNF- $\alpha$, and MCP-1 produced by LPS-induced RAW264.7 cells [75]. Intraperitoneal injection of $0.48 \mathrm{mg} / \mathrm{kg}$ can not only decrease the expression of NF- $\kappa \mathrm{B} 1$ and PKC- $\delta$ in the NF- $\kappa \mathrm{B}$ signaling pathway in the lung tissues of asthmatic mice, but also reduce the contents of IL-4, IL-10, TNF- $\alpha$, and MCP-1 in bronchoalveolar lavage fluid [76].

\section{Purine Alkaloids}

Purine alkaloids have the same purine ring structure and can be transformed into each other under specific conditions, mainly including caffeine, theophylline, theobromine, xanthine, and hypoxanthine. Among them, theophylline has a good anti-inflammatory effect and has been widely used in the clinical treatment of Chronic Obstructive Pulmonary Disease (COPD) and other pulmonary diseases.

5.1. Theophylline. Theophylline, extracted from tea and cocoa beans, has the functions of anti-inflammatory, bronchiectasis, immunity regulation, and improving the contractility of septal muscle. It has therapeutic effects on bronchial asthma, emphysema, and heart failure [77]. Theophylline exerts anti-inflammatory effects by inhibiting the release of phosphodiesterase and neuropeptides. At appropriate levels, theophylline strongly inhibits antigenactivated late-phase reactions, which are associated with the inhibition of the activation of neutrophils and the release of inflammatory mediators induced by phosphodiesterase. Theophylline has direct dilating effects on the smooth muscle of the respiratory tract [78]. Theophylline also inhibits adenosine receptor, increases the release of interleukin-10, and inhibits the transcriptional expression of NF$\kappa \mathrm{B}$ and the activation of inflammatory cells and T cells from peripheral blood to airway mucosa metastasis, thus playing an anti-inflammatory and immune regulatory role. Oral administration of $0.1 \mathrm{~g}$ of theophylline twice a day can reduce the contents of IL-4, IL-5, IL-6, IL-8, IL-17, TNF- $\alpha$, and C-reactive protein (CRP) in COPD patients. Moreover, theophylline of this dosage can increase the activity and expression of histone deacetylase- 2 in the blood of COPD patients and improve the sensitivity of the human body to glucocorticoids [79].

Moreover, a low dosage of theophylline can inhibit the abnormal expression of ET-1 induced by inflammatory cytokines in smooth muscle, which is one of its mechanisms of reducing the hyperresponsiveness of asthmatic airway.

\section{Organic Amine Alkaloids}

Organic amine alkaloid is a kind of important alkaloid. Nitrogen atoms in this alkaloid are not combined in a ring structure. Colchicine, betaine, ephedrine, demecolcine, and leonurine belong to this kind of alkaloids.

6.1. Colchicine. Colchicine, extracted from the seeds and bulbs of Colchicum autumnale L., has been now widely used in the treatment of gout and the first choice for acute gout attacks. The 2016 European rheumatic union guidelines for the treatment of gout [80] suggests that colchicine is almost equivalent to nonsteroidal anti-inflammatory drugs and 
glucocorticoids and the guidelines advocates to start the treatment of gout with low doses of colchicine.

Monosodium urate crystal deposition is the central link in the pathogenesis of gouty arthritis. When neutrophils phagocytize monosodium urate crystals, lysosomes can be induced to release a chemokine derived from glycopeptide crystals, which can significantly amplify the recruitment of neutrophils. Colchicine, by means of inhibiting the aggregation of neutrophils at the joints, weakens neutrophils' phagocytosis towards monosodium urate crystals and reduces the inflammatory response caused by partial neutrophils [81]. Moreover, colchicine can change the expression of L-selectin in neutrophils and the distribution of E-selectin in endothelial cells, so as to inhibit the release of leukotriene B4, as well as the adhesion, exudation, and recruitment of neutrophils [82]. Colchicine can inhibit not only the chemotaxis, adhesion, and superoxidation of neutrophils but also the process and release of NOD-like receptor heat protein domain-related protein inflammasomes and interleukin- $1 \beta$.

In terms of heart disease, oral administration of $1.5 \mathrm{mg}$ of colchicine for 5 days can reduce hypersensitive C-reactive protein (hs-CRP), IL-1, IL-6, IL-8, and Neutrophilic Alkaline Phosphatase 3 (NALP3) levels in patients' serum with acute myocardial infarction [83]. Moreover, it can not only improve the expression of IL-1, apoptosis-related microprotein ASC, cysteine, proteinase-caspase-1, and NLRP3 mRNA in peripheral blood but also reduce the myocardial damage mediated by the inflammatory response. Colchicine can prevent the recurrence of atrial fibrillation after radiofrequency ablation, which is closely related to the reduction of the contents of interleukin-6 and CRP. Vascular atherosclerotic disease is a chronic immune inflammatory disease and smoking is an independent risk factor for atherosclerosis disease. After the oral administration of colchicine $(0.1 \mathrm{mg} /(\mathrm{kg} \cdot \mathrm{d}))$ for eight weeks, the contents of intercellular adhesion molecule-1 (ICAM-1), vascular adhesion molecules-1 (VCAM-1), monocyte chemoattractant protein-1 (MCP-1), IL-6, IL-1 $\beta$, and TNF- $\alpha$ in the aorta of mice exposed to tobacco for a long period of time could be reduced to achieve the purpose of vascular protection [84].

\section{Indole Alkaloid}

Indole alkaloids, mainly derived from tryptophan, are the largest and most complex alkaloids. Indole alkaloids are divided into four groups: simple indoles, tryptophan indole, hemiterpenoid indoles, and monoterpene indoles.

7.1. Rhynchophylline. Rhynchophylline, a kind of monoterpene indole alkaloids, is the highest in the content of Uncaria rhynchophylla (Miq.) Miq. ex Havil. [85]. It has therapeutic effects on epilepsy, hypertension, and Parkinson's diseases. Rhynchophylline can significantly increase the pain threshold of mice and inhibit auricle swelling by reducing capillary permeability caused by xylene [86]. In vitro, $5 \mu \mathrm{g} / \mu \mathrm{L}$ of rhynchophylline can inhibit the production of IL- $1 \beta$ and TNF- $\alpha$ in dopaminergic neurons and glial cells induced by lipopolysaccharide, so as to protect neurons. Rhynchophylline can also inhibit the phosphorylation of p38 MAPK, then block the nuclear translocation of NF- $\kappa \mathrm{B}$, and inhibit its transcriptional function, so as to inhibit the vascular endothelial cell damage caused by intermittent hypoxia [87].

7.2. Brucine. Brucine, a kind of monoterpene indole alkaloids extracted from the mature seeds of Strychnos nuxvomica Linn., has therapeutic effects on myasthenia gravis, rheumatoid arthritis, and hemiplegia caused by cerebrovascular diseases [88]. Brucine can inhibit auricle swelling caused by xylene in mice. Oral administration of $3 \mathrm{mg} / \mathrm{kg}$ of brucine can inhibit adjuvant arthritis in rats and reduce the concentrations of NO and NOS in serum, thus reducing the destruction of articular cartilage and protecting the function of chondrocytes [89]. In myasthenia gravis model rats, brucine can not only reduce the serum level of acetylcholine receptor antibody and IL-6, but also inhibit the activation of $\mathrm{T}$ cells and B cells.

\section{Summary}

As people learn more and more about nature, Chinese medical herbs have become important sources for the creation of new medicines. The purpose of researches on the anti-inflammatory effects and mechanisms of alkaloids in Chinese materia medica is to find new anti-inflammatory drugs with high selectivity and strong effect and improve the effectiveness, safety, and economy of the current clinical treatment in rheumatoid arthritis, ankylosing spondylitis, and other rheumatic immune diseases.

There are many side effects of drugs currently used in the treatment of rheumatic immune diseases. Nonsteroidal antiinflammatory drugs can increase the risk of gastrointestinal bleeding. Glucocorticoids can not only raise blood sugar and blood pressure but also aggravate osteoporosis. Immunosuppressive agents can give rise to fungal or atypical pathogen infections. Alkaloids in Chinese materia medica have unique advantages in the treatment of rheumatic immune diseases. They exert anti-inflammatory effects through multiple targets, without adverse reactions mentioned above. Their anti-inflammatory mechanisms include stimulating the pituitary adrenal cortex axis, promoting the release of adrenal cortex hormones, inhibiting the release of inflammatory mediators, interleukins, and tumor necrosis factors, and regulating the level of nitric oxide, the expression of cytokine mRNA, and so on [90].

Anti-inflammatory alkaloids can be mainly divided into the following categories: isoquinoline alkaloids, indole alkaloids, pyridine alkaloids, terpenoid alkaloids, organic amine alkaloids, etc. This article summarizes anti-inflammatory mechanisms of alkaloids in Chinese materia medica, in order to provide a reference for screening active ingredients with anti-inflammatory effects and finding new therapeutic targets. However, the studies above are limited to animal experiments, and some of their mechanisms need further study. We will continue to explore the 
anti-inflammatory effects of alkaloids in Chinese materia medica in clinical practice and contribute our efforts to the development of new drugs with anti-inflammatory effects.

\section{Disclosure}

The funder has no role in the manuscript writing, editing, approval, or decision to publish.

\section{Conflicts of Interest}

The authors declare that they have no conflicts of interest.

\section{Authors' Contributions}

Sicong Li and Xin Liu contributed equally to this article. They are both responsible for the initial outline, draft writing, revisions for intellectual content, and final approval. Xiaoran Cheng and Lei Bi were responsible for data interpretation, presentation, draft writing, and revisions for intellectual content.

\section{Acknowledgments}

The authors would like to thank all the scholars who have made outstanding contributions to the internationalization of traditional Chinese medicine.

\section{References}

[1] W. Li, Y. Li, Y. Zhao, and L. Ren, "The protective effects of aloperine against ox-LDL-induced endothelial dysfunction and inflammation in HUVECs," Artificial Cells, Nanomedicine, and Biotechnology, vol. 48, no. 1, pp. 107-115, 2020.

[2] U. H. Ren Hasan, A. M. Uttra et al., "Phytochemicals targeting matrix metalloproteinases regulating tissue degradation in inflammation and rheumatoid arthritis," Phytomedicine, vol. 66, Article ID 153134, 2020.

[3] T. Wei, X. Xiaojun, and C. Peilong, "Magnoflorine improves sensitivity to doxorubicin (DOX) of breast cancer cells via inducing apoptosis and autophagy through AKT/mTOR and p38 signaling pathways," Biomedicine \& Pharmacotherapy, vol. 121, Article ID 109139, 2020.

[4] H. Ardalani, A. Hadipanah, and A. Sahebkar, "Medicinal plants in the treatment of peptic ulcer disease: a review," MiniReviews in Medicinal Chemistry, vol. 20, 2019.

[5] J. Jiang, T. Ma, L. Zhang et al., "The transdermal performance, pharmacokinetics and anti-inflammatory pharmacodynamics evaluation of harmine-loaded ethosomes," Drug Development and Industrial Pharmacy, vol. 46, no. 1, pp. 1-21, 2019.

[6] C.-J. Kim, T. W. Kim, C. J. Kim et al., "Mitochondrial dysfunction as a cause of neurological diseases," International Neurourology Journal, vol. 23, no. 1, pp. S3-S4, 2019.

[7] H. Wang, S. Tu, S. Yang et al., "Berberine modulates LPA function to inhibit the proliferation and inflammation of FLSRA via p38/ERK MAPK pathway mediated by LPA1," Evidence-Based Complementary and Alternative Medicine, vol. 2019, Article ID 2580207, 8 pages, 2019.

[8] J. Song, Q. Wu, J. Jiang et al., "Berberine reduces inflammation of human dental pulp fibroblast via miR-21/KBTBD7 axis," Archives of Oral Biology, vol. 110, Article ID 104630, 2020 .
[9] M. Yao, X. Fan, B. Yuan et al., "Berberine inhibits NLRP3 Inflammasome pathway in human triple-negative breast cancer MDA-MB-231 cell," BMC Complementary and Alternative Medicine, vol. 19, no. 1, p. 216, 2019.

[10] Z.-C. Yu, Y.-X. Cen, B.-H. Wu et al., "Berberine prevents stress-induced gut inflammation and visceral hypersensitivity and reduces intestinal motility in rats," World Journal of Gastroenterology, vol. 25, no. 29, pp. 3956-3971, 2019.

[11] M. Takahara, A. Takaki, S. Hiraoka et al., "Berberine improved experimental chronic colitis by regulating interferon-gammaand IL-17A-producing lamina propria CD4(+) T cells through AMPK activation," Science Reports, vol. 9, no. 1, p. 11934, 2019.

[12] W. Zhang, J.-H. Xu, T. Yu, and Q.-K. Chen, "Effects of berberine and metformin on intestinal inflammation and gut microbiome composition in $\mathrm{db} / \mathrm{db}$ mice," Biomedicine \& Pharmacotherapy, vol. 118, Article ID 109131, 2019.

[13] K. Chen, Q. Chen, N. Wu et al., "Berberine ameliorates spatial learning memory impairment and modulates cholinergic anti-inflammatory pathway in diabetic rats," Frontiers in Pharmacology, vol. 10, p. 1003, 2019.

[14] M. Beba, K. Djafarian, and S. Shab-Bidar, "Effect of Berberine on C-reactive protein: a systematic review and meta-analysis of randomized controlled trials," Complementary Therapies in Medicine, vol. 46, pp. 81-86, 2019.

[15] Y. Jia, Y. Miao, M. Yue, M. Shu, Z. Wei, and Y. Dai, “Tetrandrine attenuates the bone erosion in collagen-induced arthritis rats by inhibiting osteoclastogenesis via spleen tyrosine kinase," The FASEB Journal, vol. 32, no. 6, pp. 33983410, 2018.

[16] G. Qin, B. Gui, J. Xie et al., "Tetrandrine alleviates nociception in a rat model of migraine via suppressing S100B and p-ERK activation in satellite glial cells of the trigeminal Ganglia," Journal of Molecular Neuroscience, vol. 64, no. 1, pp. 29-38, 2018.

[17] N. Bhagya and K. R. Chandrashekar, "Tetrandrine-a molecule of wide bioactivity," Phytochemistry, vol. 125, pp. 5-13, 2016.

[18] L.-N. Gao, Q.-S. Feng, X.-F. Zhang, Q.-S. Wang, and Y.-L. Cui, "Tetrandrine suppresses articular inflammatory response by inhibiting pro-inflammatory factors via NF- $\kappa \mathrm{B}$ inactivation," Journal of Orthopaedic Research, vol. 34, no. 9, pp. 1557-1568, 2016.

[19] X. Yuan, B. Tong, Y. Dou, X. Wu, Z. Wei, and Y. Dai, "Tetrandrine ameliorates collagen-induced arthritis in mice by restoring the balance between Th17 and Treg cells via the aryl hydrocarbon receptor," Biochemical Pharmacology, vol. 101, pp. 87-99, 2016.

[20] G. Bao, C. Li, L. Qi, N. Wang, and B. He, "Tetrandrine protects against oxygen-glucose-serum deprivation/reoxygenationinduced injury via PI3K/AKT/NF- $\kappa \mathrm{B}$ signaling pathway in rat spinal cord astrocytes," Biomedicine \& Pharmacotherapy, vol. 84, pp. 925-930, 2016.

[21] B. Qiao, H. Wang, C. Wang, M. Liang, K. Huang, and Y. Li, "Dauricine negatively regulates lipopolysaccharide- or cecal ligation and puncture-induced inflammatory response via NF- $\kappa \mathrm{B}$ inactivation," Archives of Biochemistry and Biophysics, vol. 666, pp. 99-106, 2019.

[22] Z. Pu, S. Ma, L. Wang et al., "Corrigendum to "Amyloid-beta degradation and neuroprotection of dauricine mediated by unfolded protein response in a Caenorhabditis elegans model of Alzheimer's disease" [Neuroscience 392C (2018) 25-37]," Neuroscience, vol. 404, p. 557, 2019. 
[23] X. Zhou, Y. Q. Qu, Z. Zheng et al., "Novel dauricine derivatives suppress cancer via autophagy-dependent cell death," Bioorganic Chemistry, vol. 83, pp. 450-460, 2019.

[24] H. Li, X. Chen, and S.-J. Zhou, "Dauricine combined with clindamycin inhibits severe pneumonia co-infected by influenza virus $\mathrm{H} 5 \mathrm{~N} 1$ and Streptococcus pneumoniae in vitro and in vivo through NF- $\kappa \mathrm{B}$ signaling pathway," Journal of Pharmacological Sciences, vol. 137, no. 1, pp. 12-19, 2018.

[25] S. Y. Zhang and J. Q. Ren, "Dauricine inhibits viability and induces cell cycle arrest and apoptosis via inhibiting the PI3K/ Akt signaling pathway in renal cell carcinoma cells," Molecular Medicine Reports, vol. 17, no. 5, pp. 7403-7408, 2018.

[26] M.Y. Wu, S. F. Wang, C. Z. Cai et al., "Natural autophagy blockers, dauricine (DAC) and daurisoline (DAS), sensitize cancer cells to camptothecin-induced toxicity," Oncotarget, vol. 8, no. 44, pp. 77673-77684, 2017.

[27] H. Jin, S. Shen, X. Chen, D. Zhong, and J. Zheng, "CYP3Amediated apoptosis of dauricine in cultured human bronchial epithelial cells and in lungs of CD-1 mice," Toxicology and Applied Pharmacology, vol. 261, no. 3, pp. 248-254, 2012.

[28] H. Yang, J. Wang, X. Chen et al., "Effects of sinomenine in LPS-associated diseases are related to inhibition of LBP, Mac1, and L-selectin levels," Journal of Veterinary Pharmacology and Therapeutics, vol. 42, no. 6, pp. 732-737, 2019.

[29] L. Zhang, W. Zhang, B. Zheng, and N. Tian, "Sinomenine attenuates traumatic spinal cord injury by suppressing oxidative stress and inflammation via Nrf2 pathway," Neurochemical Research, vol. 44, no. 4, pp. 763-775, 2019.

[30] R.-L. Tian, Y.-K. Zhi, L. Yi et al., "Sinomenine regulates CD14/ TLR4, JAK2/STAT3 pathway and calcium signal via $\alpha 7 \mathrm{nAChR}$ to inhibit inflammation in LPS-stimulated macrophages," Immunopharmacology and Immunotoxicology, vol. 41, no. 1, pp. 172-177, 2019.

[31] Z.-t. Feng, T. Yang, X.-q. Hou et al., "Sinomenine mitigates collagen-induced arthritis mice by inhibiting angiogenesis," Biomedicine \& Pharmacotherapy, vol. 113, Article ID 108759, 2019.

[32] Y. Liu, Y. Sun, Y. Zhou et al., "Sinomenine hydrochloride inhibits the progression of plasma cell mastitis by regulating IL-6/JAK2/STAT3 pathway," International Immunopharmacology, Article ID 106025, 2019.

[33] W. Wang, X. Yang, Q. Chen et al., "Sinomenine attenuates septic-associated lung injury through the Nrf2-Keap1 and autophagy," Journal of Pharmacy and Pharmacology, vol. 72, no. 2, pp. 259-270, 2020.

[34] B. Gao, Y. Wu, Y. J. Yang et al., "Sinomenine exerts anticonvulsant profile and neuroprotective activity in pentylenetetrazole kindled rats: involvement of inhibition of NLRP1 inflammasome," Journal of Neuroinflammation, vol. 15, no. 1, p. 152, 2018.

[35] L. Ning, S. Wan, Z. Jie et al., "Lycorine induces apoptosis and G1 phase Arrest through ROS/p38 MAPK signaling pathway in human osteosarcoma cells in vitro and in vivo," Spine, vol. 45, no. 3, pp. 126-139, 2020.

[36] W. Liu, Q. Zhang, Q. Tang et al., "[Corrigendum] Lycorine inhibits cell proliferation and migration by inhibiting ROCK1/cofilininduced actin dynamics in HepG2 hepatoblastoma cells," Oncology Reports, vol. 42, no. 6, p. 2856, 2019.

[37] H. J. Park, M. Gholam-Zadeh, J. H. Suh et al., "Lycorine attenuates autophagy in osteoclasts via an axis of mROS/ TRPML1/TFEB to reduce LPS-induced bone loss," Oxidative Medicine and Cellular Longevity, vol. 2019, Article ID 8982147, 11 pages, 2019.

[38] L. Yang, J. H. Zhang, X. L. Zhang et al., "Tandem mass tagbased quantitative proteomic analysis of lycorine treatment in highly pathogenic avian influenza H5N1 virus infection," PeerJ, vol. 7, Article ID e7697, 2019.

[39] H. Hu, S. Wang, D. Shi et al., "Lycorine exerts antitumor activity against osteosarcoma cells in vitro and in vivo xenograft model through the JAK2/STAT3 pathway," OncoTargets and Therapy, vol. 12, pp. 5377-5388, 2019.

[40] P. M. Le, V. Srivastava, T. T. Nguyen et al., "Stephanine from Stephania venosa (blume) spreng showed effective antiplasmodial and anticancer activities, the latter by inducing apoptosis through the reverse of mitotic exit," Phytotherapy Research, vol. 31, no. 9, pp. 1357-1368, 2017.

[41] H. Wang, X. Cheng, S. Kong et al., "Synthesis and structureactivity relationships of a series of Aporphine derivatives with antiarrhythmic activities and acute toxicity," Molecules, vol. 21 , no. 12, pp. 22-24, 2016.

[42] C. Desgrouas, N. Taudon, S.-S. Bun et al., "Ethnobotany, phytochemistry and pharmacology of Stephania rotunda Lour," Journal of Ethnopharmacology, vol. 154, no. 3, pp. 537-563, 2014.

[43] B. Baghdikian, V. Mahiou-Leddet, S. Bory et al., "New antiplasmodial alkaloids from Stephania rotunda," Journal of Ethnopharmacology, vol. 145, no. 1, pp. 381-385, 2013.

[44] H. Dong, Y. Zhang, L. Fang et al., "Combinative application of $\mathrm{pH}$-zone-refining and conventional high-speed countercurrent chromatography for preparative separation of alkaloids from Stephania kwangsiensis," Journal of Chromatography B, vol. 879, no. 13-14, pp. 945-949, 2011.

[45] Z. Ren, X. Wang, X. Chen et al., "Identification of Aloperine as an anti-apoptotic Bcl2 protein inhibitor in glioma cells," PeerJ, vol. 7, Article ID e7652, 2019.

[46] Q. Mao, F. Guo, X. Liang et al., "Aloperine Activates the PI3K/ Akt pathway and protects against coronary microembolisation-induced myocardial injury in rats," Pharmacology, vol. 104, no. 1-2, pp. 90-97, 2019.

[47] H. I. Yu, H. C. Shen, S. H. Chen et al., "Autophagy modulation in human thyroid cancer cells following aloperine treatment," International Journal of Molecular Sciences, vol. 20, no. 21, 2019.

[48] J.-S. Liu, C.-Y. Huo, H.-H. Cao et al., "Aloperine induces apoptosis and G2/M cell cycle arrest in hepatocellular carcinoma cells through the PI3K/Akt signaling pathway," Phytomedicine, vol. 61, Article ID 152843, 2019.

[49] Z. Chang, P. Zhang, M. Zhang et al., "Aloperine suppresses human pulmonary vascular smooth muscle cell proliferation via inhibiting inflammatory response," Chinese Journal of Physiology, vol. 62, no. 4, pp. 157-165, 2019.

[50] N. Zhang, Y. Dou, L. Liu et al., "SA-49, a novel aloperine derivative, induces MITF-dependent lysosomal degradation of PD-L1," EBioMedicine, vol. 40, pp. 151-162, 2019.

[51] D. Tian, Y. Li, X. Li et al., "Aloperine inhibits proliferation, migration and invasion and induces apoptosis by blocking the Ras signaling pathway in human breast cancer cells," Molecular Medicine Reports, vol. 18, no. 4, pp. 3699-3710, 2018.

[52] H. Zhuang, X. Dai, X. Zhang, Z. Mao, and H. Huang, "Sophoridine suppresses macrophage-mediated immunosuppression through TLR4/IRF3 pathway and subsequently upregulates $\mathrm{CD}^{+}$T cytotoxic function against gastric cancer," Biomedicine \& Pharmacotherapy, vol. 121, Article ID 109636, 2020.

[53] R. Wang, H. Liu, Y. Shao et al., "Sophoridine inhibits human colorectal cancer progression via targeting MAPKAPK2," Molecular Cancer Research, vol. 17, no. 12, pp. 2469-2479, 2019. 
[54] G. Ren, G. Ding, H. Zhang et al., "Antiviral activity of sophoridine against enterovirus 71 in vitro," Journal of Ethnopharmacology, vol. 236, pp. 124-128, 2019.

[55] X. D. Chen, X. Y. Hua, X. M. Kong et al., "[Sophoridine inhibits the proliferation of human gastric cancer MKN45 cells and promotes apoptosis]," Sheng Li Xue Bao, vol. 70, no. 4, pp. 391-396, 2018.

[56] D. Li, L. Dai, X. Zhao et al., "Novel sophoridine derivatives bearing phosphoramide mustard moiety exhibit potent antitumor activities in vitro and in vivo," Molecules, vol. 23, no. 8, 2018.

[57] Z. Yue, T. Si, Z. Pan et al., "Sophoridine suppresses cell growth in human medulloblastoma through FoxM1, NF-kappaB and AP-1," Oncology Letters, vol. 14, no. 6, pp. 7941-7946, 2017.

[58] S. Zhang, S. Guo, X. b. Gao et al., "Matrine attenuates high-fat diet-induced in vivo and ox-LDL-induced in vitro vascular injury by regulating the PKC $\alpha /$ eNOS and PI3K/Akt/eNOS pathways," Journal of Cellular and Molecular Medicine, vol. 23, no. 4, pp. 2731-2743, 2019.

[59] P. Li, J. Lei, G. Hu et al., "Matrine mediates inflammatory response via gut microbiota in TNBS-induced murine colitis," Frontiers in Physiology, vol. 10, p. 28, 2019.

[60] X. Yu, H. J. Seow, H. Wang et al., "Matrine reduces cigarette smoke-induced airway neutrophilic inflammation by enhancing neutrophil apoptosis," Clinical Science, vol. 133, no. 4, pp. 551-564, 2019.

[61] W. W. Li, T. Y. Wang, B. Cao et al., "Synergistic protection of matrine and lycopene against lipopolysaccharideinduced acute lung injury in mice," Molecular Medicine Reports, vol. 20, no. 1, pp. 455-462, 2019.

[62] J. Zhou, W. Ma, X. Wang et al., "Matrine suppresses reactive oxygen species (ROS)-Mediated MKKs/p38-induced inflammation in oxidized low-density lipoprotein (ox-LDL)Stimulated macrophages," Medical Science Monitor, vol. 25, pp. 4130-4136, 2019.

[63] Q. Li, J. Xu, Z. He et al., "The effects of matrine in combination with docetaxel on castration-resistant (Androgen-Independent) prostate cancer," Cancer Management and Research, vol. 11, pp. 10125-10133, 2019

[64] W.-W. Jiang, Y.-M. Wang, X.-Y. Wang, Q. Zhang, S.-M. Zhu, and C.-L. Zhang, "Role and mechanism of matrine alone and combined with acitretin for $\mathrm{HaCaT}$ cells and psoriasis-like murine models," Chinese Medical Journal, vol. 132, no. 17, pp. 2079-2088, 2019.

[65] C. Wenjin and W. Jianwei, "Protective effect of gentianine, a compound from du huo Ji sheng tang, against Freund's complete adjuvant-induced arthritis in rats," Inflammation, vol. 40, no. 4, pp. 1401-1408, 2017.

[66] G. O. Anyanwu, Nisar-ur-Rehman, C. E. Onyeneke, and K. Rauf, "Medicinal plants of the genus Anthocleista-a review of their ethnobotany, phytochemistry and pharmacology," Journal of Ethnopharmacology, vol. 175, pp. 648-667, 2015.

[67] X. Rauf, S. Tang, Y. Jin et al., "Determination of the metabolic profile of gentianine after oral administration to rats by high performance liquid chromatography/electrospray ionizationtrap mass spectrometry," Journal of Chromatography B, vol. 989, pp. 98-103, 2015.

[68] S. H. Tiong, C. Y. Looi, A. Arya et al., "Vindogentianine, a hypoglycemic alkaloid from Catharanthus roseus (L.) G. Don (Apocynaceae)," Fitoterapia, vol. 102, pp. 182-188, 2015.

[69] H. Vaidya, R. K. Goyal, and S. K. Cheema, "Anti-diabetic activity of swertiamarin is due to an active metabolite, gentianine, that upregulates PPAR- $\gamma$ gene expression in 3T3-L1 cells," Phytotherapy Research, vol. 27, no. 4, pp. 624-627, 2013.
[70] F. Peng, N. Zhang, C. Wang et al., "Aconitine induces cardiomyocyte damage by mitigating BNIP3-dependent mitophagy and the TNFalpha-NLRP3 signalling axis," Cell Proliferation, vol. 53, no. 1, Article ID e12701, 2020.

[71] T. Guo, N. Cheng, J. Zhao, X. Hou, Y. Zhang, and N. Feng, "Novel nanostructured lipid carriers-loaded dissolving microneedles for controlled local administration of aconitine," International Journal of Pharmaceutics, vol. 572, Article ID 118741, 2019.

[72] Y. Wang, Y. Shan, Y. Wang et al., "Aconitine inhibits androgen synthesis enzymes by rat immature Leydig cells via down-regulating androgen synthetic enzyme expression in vitro," Chemico-Biological Interactions, vol. 312, Article ID 108817, 2019

[73] X. Zhan, W. Zhang, T. Sun et al., "Bulleyaconitine A effectively relieves allergic lung inflammation in a murine asthmatic model," Medical Science Monitor, vol. 25, pp. 1656-1662, 2019.

[74] M. X. Xie, H. Q. Zhu, R. P. Pang et al., "Mechanisms for therapeutic effect of bulleyaconitine A on chronic pain," Molecular Pain, vol. 14, Article ID 1744806918797243, 2018.

[75] M. X. Xie, J. Yang, R. P. Pang et al., "Bulleyaconitine A attenuates hyperexcitability of dorsal root ganglion neurons induced by spared nerve injury: the role of preferably blocking Nav1.7 and Nav1.3 channels," Molecular Pain, vol. 14, Article ID 1744806918778491, 2018.

[76] M.-X. Xie, R.-P. Pang, J. Yang et al., "Bulleyaconitine A preferably reduces tetrodotoxin-sensitive sodium current in uninjured dorsal root ganglion neurons of neuropathic rats probably via inhibition of protein kinase C," Pain, vol. 158, no. 11, pp. 2169-2180, 2017.

[77] Y. Bin, Y. Xiao, D. Huang et al., "Theophylline inhibits cigarette smoke-induced inflammation in skeletal muscle by upregulating HDAC2 expression and decreasing NF- $\kappa \mathrm{B}$ activation," American Journal of Physiology-Lung Cellular and Molecular Physiology, vol. 316, no. 1, pp. L197-L205, 2019.

[78] T. Mitani, T. Takaya, N. Harada et al., "Theophylline suppresses interleukin-6 expression by inhibiting glucocorticoid receptor signaling in pre-adipocytes," Archives of Biochemistry and Biophysics, vol. 646, pp. 98-106, 2018.

[79] L. Gallelli, D. Falcone, R. Cannataro et al., "Theophylline action on primary human bronchial epithelial cells under proinflammatory stimuli and steroidal drugs: a therapeutic rationale approach," Drug Design, Development and Therapy, vol. 11, pp. 265-272, 2017.

[80] P. Richette, M. Doherty, E. Pascual et al., "2016 updated EULAR evidence-based recommendations for the management of gout," Annals of the Rheumatic Diseases, vol. 76, no. 1, pp. 29-42, 2017.

[81] N. Saini, D. Singh, and R. Sandhir, "Bacopa monnieri prevents colchicine-induced dementia by anti-inflammatory action," Metabolic Brain Disease, vol. 34, no. 2, pp. 505-518, 2019.

[82] A. Berdeli, O. Senol, and G. Talay, "Treatment of familial mediterranean fever with canakinumab in patients who are unresponsive to colchicine," European Journal of Rheumatology, vol. 6, no. 2, pp. 85-88, 2019.

[83] B. Tucker, R. Kurup, J. Barraclough et al., "Colchicine as a novel therapy for suppressing chemokine production in patients with an acute coronary syndrome: a pilot study," Clinical Therapeutics, vol. 41, no. 10, pp. 2172-2181, 2019.

[84] Y. Liang, H.-F. Zhou, M. Tong, L. Chen, K. Ren, and G.-J. Zhao, "Colchicine inhibits endothelial inflammation via NLRP3/CRP pathway," International Journal of Cardiology, vol. 294, p. 55, 2019. 
[85] L. Hongyan, Z. Mengjiao, W. Chunyan, and H. Yaruo, "Rhynchophylline attenuates neurotoxicity in tourette syndrome rats," Neurotoxicity Research, vol. 36, no. 4, pp. 679687, 2019.

[86] Y. Yaruo, J. Sun, S. Zhu et al., “The role of rhynchophylline in alleviating early brain injury following subarachnoid hemorrhage in rats," Brain Research, vol. 1631, pp. 92-100, 2016.

[87] H. C. Hsu, N. Y. Tang, C. H. Liu et al., "Antiepileptic effect of Uncaria rhynchophylla and rhynchophylline involved in the initiation of c-Jun $\mathrm{N}$-terminal kinase phosphorylation of MAPK signal pathways in acute seizures of Kainic acidtreated rats," Evidence-Based Complementary and Alternative Medicine, vol. 2013, Article ID 961289, 2013.

[88] M. Zhang, C. Wang, H.-l. Cai, J. Wen, and P.-f. Fang, "Licorice extracts attenuate nephrotoxicity induced by brucine through suppression of mitochondria apoptotic pathway and STAT3 activation," Current Medical Science, vol. 39, no. 6, pp. 890-898, 2019.

[89] Z. P. Chen, W. Liu, H. X. Chen et al., "[Brucine chitosan thermosensitive hydrogel for intra-articular injection]," Yao Xue Xue Bao, vol. 47, no. 5, pp. 652-656, 2012.

[90] H. S. Foyet, W. E. Keugong, A. H. Ngatanko et al., "Anticholinesterase and antioxidant potential of hydromethanolic extract of Ziziphus mucronata (rhamnaceae) leaves on scopolamine-induced memory and cognitive dysfunctions in mice," Evidence-Based Complementary and Alternative Medicine, vol. 2019, Article ID 4568401, 2019. 